\title{
An Atomic Level Study of Localized Strain Fields on Multiple Low-Index Ceria $\left(\mathrm{CeO}_{2}\right)$ Nanoparticle Surfaces
}

Piyush Haluai ${ }^{1}$, Ethan L. Lawrence ${ }^{1}$, Tara Boland ${ }^{2}$ and Peter Crozier ${ }^{3}$

${ }^{1}$ Arizona State University, United States, ${ }^{2}$ Arizona State University, Arizona, United States, ${ }^{3}$ Arizona State University, Tempe, Arizona, United States

Surface strain engineering has emerged as a very promising field for modifying/ altering the structureproperty relationships in materials at atomic level [1]. With increasing demand for higher efficiency, strain-tuning at the surface has gained a lot of attention in recent times. For example, surface strain can regulate and control surface diffusion processes and can change functionalities in chemical processes such as, enhancement of oxygen reduction activity [2][3], strain tunable catalytic properties [4][5][6] etc. Ceria is a commonly used material for energy-based applications such as catalytic converter of cars, electrode for the solid oxide fuel cells, etc. The degree of strain can be tuned by different means such as, particle size, shape, non-stoichiometry (e.g., oxygen vacancies), ambient environment (e.g., oxygen atmosphere, vacuum) etc.

We have studied the surface strain in reducible oxide nanoparticles $\left(\mathrm{CeO}_{2}\right)$ with atomic resolution using high resolution transmission electron microscopy (HRTEM). In a typical TEM image, the signal from the heavier $\mathrm{Ce}$ atomic columns is much stronger than the signal from the lighter oxygen columns. Consequently, more precise measurements can be made on the cation sublattice in order to map the strain on or near the nanoparticle surface.

$\mathrm{CeO}_{2}$ nanoparticles were synthesized by the hydrothermal method [7] and imaged using negative Cs imaging in a FEI Titan AC-ETEM with a single-electron-detection K2 camera operated in the counting mode. The $\mathrm{K} 2$ camera allowed high quality electron imaging to be performed. (111) and (100) $\mathrm{CeO}_{2}$ nanoparticle surfaces were imaged in a [110] projection at $5000 \mathrm{e}-\AA$ - $-2 \mathrm{~s}-1$ with $\mathrm{Ce}$ and $\mathrm{O}$ atomic columns visible at the surface (figure 1). Custom written MATLAB codes are used to identify and determine the positions of atomic columns by fitting a two dimensional (2D) elliptical Gaussian to each Ce column with picoscale precision [8]. Atomic resolution strain maps were created along different crystallographic directions to visualize cation sublattice distortions at different locations in the nanoparticle. The strain is calculated based on a relative change of cation sublattice at the surface of the nanoparticle as compared to the bulk. In vacuum environment inside the microscope, the bulk of the nanoparticle is relatively strain free, but the surfaces show varying degrees of compressive and tensile strain along different crystallographic directions of the nanoparticle (figure $2 b$ ). The outer plane directions show highly tensile component of the strain field and is found to be originating from outward relaxation of these surfaces as backed up by Molecular Dynamics (MD) simulations (figure 2a). The highest degree of strain is associated with defects such as step sites. The in-plane strain fields on $\mathrm{CeO}_{2}$ nanoparticle surfaces are correlated with local activity for oxygen vacancy creation and annihilation. We are currently exploring the changes in the strain field with varying electron dose inside a TEM. 


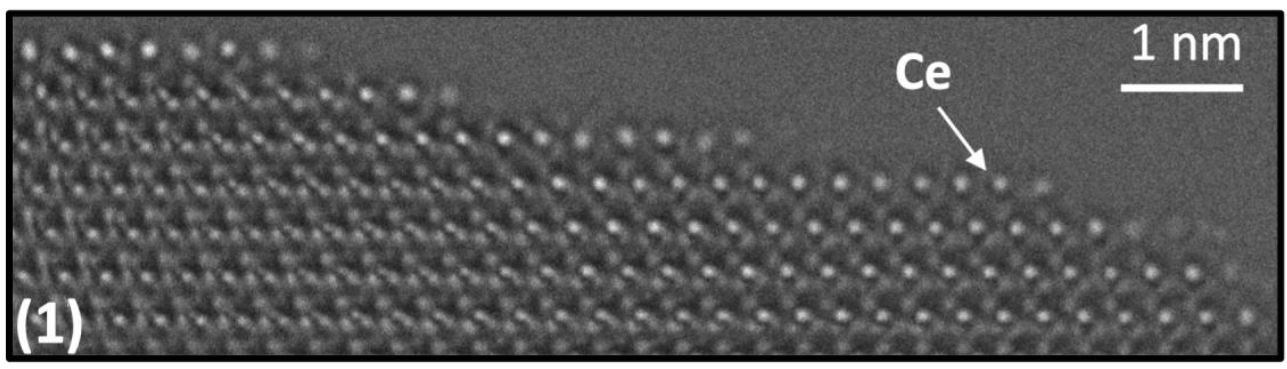

Figure 1. Figure 1: Ceria nanoparticle imaged in [110] projection. The Ce atomic column positions appear as bright dots due to negative Cs imaging (marked by arrow).

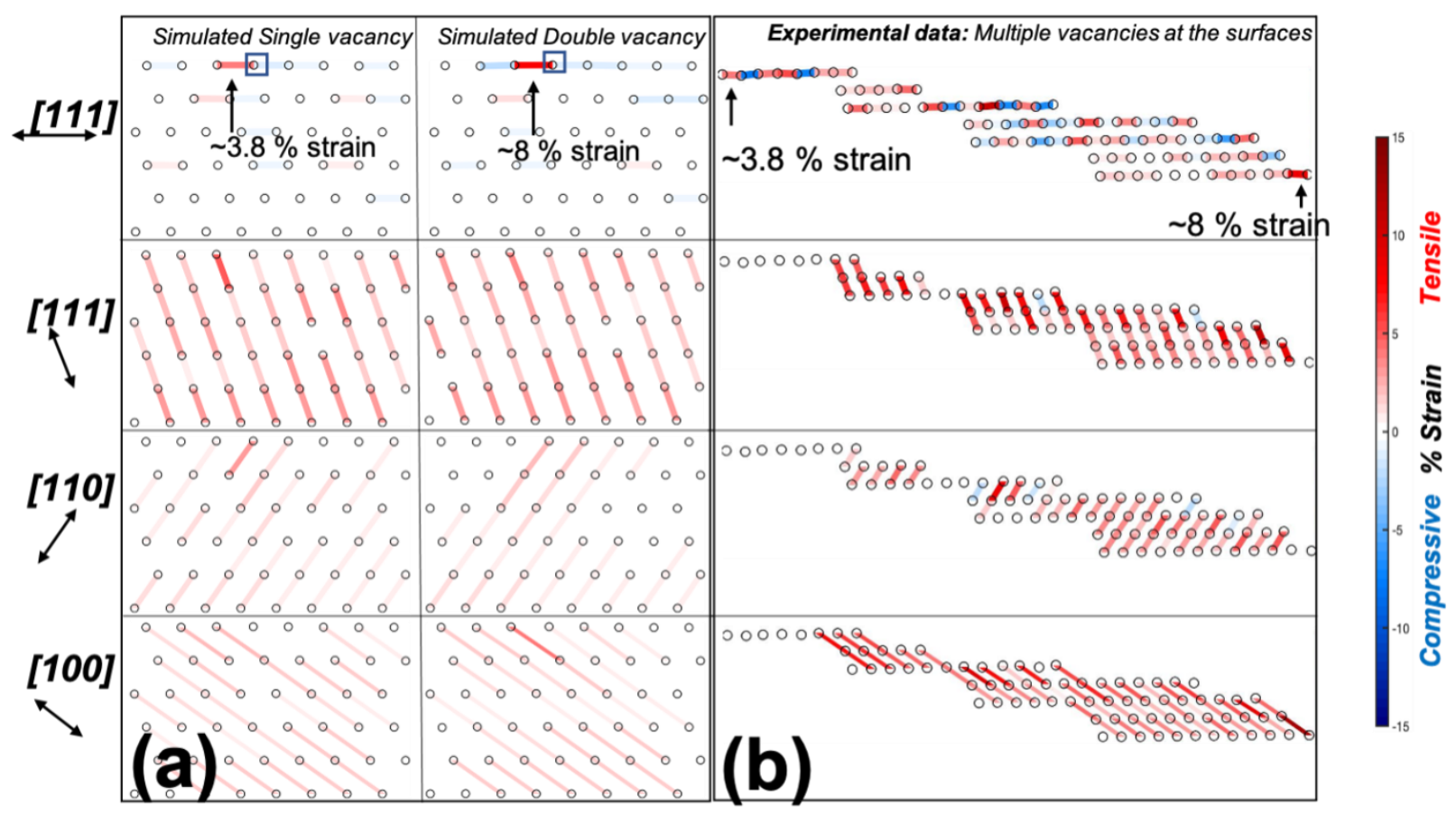

Figure 2. Figure 2: (a) Strainmaps of the simulated single and double vacancy (indicated by the box) structures at the surface of $\mathrm{CeO} 2$ nanoparticle. (b) Experimental data showing varying strain fields between atomic columns along 4 different crystallographic directions (indicated at the left of the image). The Simulated structures are relaxed using MD simulations after creation of the oxygen vacancies. The matching \%Strain between simulation and Experiment are indicated by the arrows.

References

[1] J. Madsen, P. Liu, J. B. Wagner, T. W. Hansen, and J. Schiøtz, "Accuracy of surface strain measurements from transmission electron microscopy images of nanoparticles," Adv. Struct. Chem. Imaging, vol. 3, no. 1, p. 14, Oct. 2017.

[2] S. E. Temmel, E. Fabbri, D. Pergolesi, T. Lippert, and T. J. Schmidt, "Investigating the Role of Strain toward the Oxygen Reduction Activity on Model Thin Film Pt Catalysts," ACS Catal., vol. 6, no. 11, pp. 7566-7576, Nov. 2016.

[3] J. Wu, L. Qi, H. You, A. Gross, J. Li, and H. Yang, "Icosahedral Platinum Alloy Nanocrystals with Enhanced Electrocatalytic Activities,” J. Am. Chem. Soc., vol. 134, no. 29, pp. 11880-11883, Jul. 2012. 
[4] T. Nilsson Pingel, M. Jørgensen, A. B. Yankovich, H. Grönbeck, and E. Olsson, "Influence of atomic site-specific strain on catalytic activity of supported nanoparticles," Nat. Commun., vol. 9, no. 1, Art. no. 1, Jul. 2018.

[5] F. Li et al., "Impact of Strain-Induced Changes in Defect Chemistry on Catalytic Activity of Nd2NiO4+ $\delta$ Electrodes," ACS Appl. Mater. Interfaces, vol. 10, no. 43, pp. 36926-36932, Oct. 2018.

[6] T. X. T. Sayleet al., "Strain and Architecture-Tuned Reactivity in Ceria Nanostructures; Enhanced

Catalytic Oxidation of CO to CO 2," Chem. Mater., vol. 24, no. 10, pp. 1811-1821, May 2012.

[7] Z. Yang, K. Zhou, X. Liu, Q. Tian, D. Lu, and S. Yang, "Single-crystalline ceria nanocubes: sizecontrolled synthesis, characterization and redox property," Nanotechnology, vol. 18, no. 18, p. 185606, Apr. 2007.

[8] B. D. A. Levin, E. L. Lawrence, and P. A. Crozier, "Tracking the picoscale spatial motion of atomic columns during dynamic structural change," Ultramicroscopy, vol. 213, p. 112978, Jun. 2020.

[9] We gratefully acknowledge support of NSF grant DMR-1308085, DOE BES DE-SC0004954, the use of ASU's John M. Cowley Center for High Resolution Electron Microscopy and use of the K2 IS camera courtesy of Gatan. 